\title{
LES MOTS DU CORPS : LES CHEVEUX
}

\author{
Alicja KACPRZAK
}

Université de Łódź

\begin{abstract}
En): Given the special importance of hair as a part of the human body, our article first presents a brief reflection on how the word hair is defined in different dictionaries. We also tackle the question of its syntagmatic combinations, including the freezes in which it appears. The semantics of this lexeme is analyzed from the point of view of its polysemy, testifying to the cognitive importance of the concept itself, then of synonymy. This last approach leads us to note the existence of diastratic equivalents of the word hair (x), falling under different non-standard variants.
\end{abstract}

Keywords (En): linguistic variation; lexical variants; standard variant; phrase combinations; semantic values

Mots-clés (Fr) : variation linguistique ; variantes lexicales ; variante standard ; combinaisons syntagmatiques; valeurs sémantiques

DOI : $10.32725 /$ eer.2021.008

\section{Ô toison, moutonnant jusque sur l'encolure!} Ô boucles !

\section{Introduction}

Les cheveux, cet élément du corps humain qui trouve de très nombreuses représentations dans l'art, constituent aussi un motif récurrent dans la littérature (la Chevelure, poème de Charles Baudelaire en offre un exemple) et la mythologie, ce qui reflète sans doute une valeur particulière qui leur est attachée dans la tradition culturelle en général. Diverses significations symboliques évoquées par les cheveux sont profondément enracinées dans les croyances et les religions. Yves Mirande écrit à ce propos :

La chevelure et les cheveux ont toujours été présents dans l'histoire et dans la mythologie. Chez tous les peuples anciens, les cheveux ont été l'objet d'un culte. Homère, Hésiode, la Bible, les Védas indiens, les poèmes islandais, les chants celtiques font l'éloge des belles chevelures. Véritable parure, la chevelure est, depuis les temps les plus reculés, considérée chez l'homme comme un symbole de force et de puissance.

(MIRANDE, 2008)

Selon le récit biblique de Samson et de Dalila, le rasage de sept tresses de la chevelure de Samson, pendant qu'il dormait sur les genoux de Dalila, l'a privé de sa force et en même temps du secours de Dieu. Le symbolisme du rasage forcé

\footnotetext{
${ }^{1}$ Baudelaire Charles (1861), poème La chevelure, section « Spleen et idéal », Fleurs du mal.
} 
semble d'ailleurs profondément négatif de tout temps, connotant servitude et/ou dégradation.

Depuis l'Antiquité, les esclaves étaient rasés. Jules César faisait raser les Gaulois vaincus avant de les envoyer à Rome comme esclaves. À l'époque mérovingienne, tondre un roi c'était lui enlever sa puissance virile et son droit à régner (loi salique).

(DARDOUR, 1990)

Ce même geste d'humiliation était appliqué dans différents pays européens pendant et après la Deuxième Guerre mondiale à des femmes ayant entretenu des relations avec des occupants allemands. Or chez la femme, le symbolisme des cheveux vus comme un gage de féminité porte essentiellement sur la puissance de la séduction : par conséquent certaines communautés culturelles interdisent à la femme d'exposer sa chevelure en public. Selon Jean-Louis Chrétien : « Le voile qui la recouvre chez les femmes en certaines sociétés est la reconnaissance directe de cette puissance. » (CHRÉTIEN, 2005 :183). Dans ce cas, la valeur symbolique des cheveux s'accompagne sans doute de celle où ils sont vus comme parure, souvent au même titre que le vêtement.

Selon Jean-Claude Dardour, ces deux caractères des cheveux se distinguent par leur pérennité, car le symbolisme du cheveu «a toujours été le même à travers les âges et les pays ", alors que l'aspect du cheveu parure « est variable avec les époques et les pays » (DARDOUR, 1990).

En linguistique, la problématique du cheveu appelle encore d'autres résolutions, vu la question fondamentale de la signification du mot même, abordée notamment sous l'angle de la typologie lexicale synchronique. Peter Koch inscrit ce problème dans la thématique des hiérarchies conceptuelles en français en contraste avec d'autres langues, liée à celle du découpage linguistique, autonome dans toute langue :

L'aspect hiérarchique concerne, entre autres, des questions que la linguistique discute depuis longtemps en termes de "découpage » linguistique de la réalité extralinguistique. Pourquoi le français distingue-t-il cheveu et poil, là où d'autres langues, comme l'allemand, n'ont qu'un seul terme (Haar) ou encore pourquoi d'autres ont-elles un découpage plus fin ? (КоCH, 2005 : 12-13)

En effet, dans certaines langues, notamment en polonais, une différenciation nécessaire se dessine entre les lexies désignant la pilosité humaine en général, aussi bien sur le crâne que sur le corps (włosy) et la pilosité animale (sierść). Par contre, comme le précise Koch dans le même article (2006 : 16), le français fait partie d'un groupe de langues (italien, hongrois, japonais et autres) dans lesquelles une différenciation intervient entre d'un côté les mots renvoyant à la pilosité du crâne (cheveux) et la pilosité du reste du corps humain ainsi que du corps des animaux (poils).

Prise en compte la distinction observée par le français, notre article n'abordera que le thème de la production filiforme poussant sur le crâne humain, désignée par le mot cheveu, voire plus souvent cheveux, car se rapportant à l'ensemble de cheveux formant une chevelure. Après une brève réflexion sur la manière dont le mot cheveu est défini dans des dictionnaires, nous nous intéresserons en premier 
lieu à sa syntagmatique, vue sous l'angle de ses combinaisons adjectivales et verbales, ainsi que du point de vue des figements où il apparaît. En second lieu, nous nous pencherons sur la sémantique de cette lexie, analysée d'abord selon le point de vue de sa polysémie témoignant de l'importance cognitive du concept même, ensuite de la synonymie qui la concerne. Cette dernière approche nous mènera à découvrir les équivalents diastratiques du mot cheveu(x), relevant de différentes variantes non standard. Notre étude sera basée sur des données relatives à cheveu provenant des dictionnaires de français standard et argotiques (qui seront indiqués au fur et à mesure), ainsi que de la base Frantext.

\section{Les définitions du mot cheveu/cheveux}

Il est significatif que, même si entre le mot cheveu et le mot poil il y a une opposition sémantique nécessaire, les définitions dictionnairiques du premier d'entre eux recourent paradoxalement au deuxième qui est ainsi employé à titre d'hyperonyme.

Ainsi, trouvons-nous dans le dictionnaire du CNRTL et dans le Larousse les définitions suivantes :

Cheveu : A.- Gén. au plur. Poil qui pousse sur le crâne de l'homme².

Cheveu : Chacun des poils qui poussent sur la tête, chez les humains; ensemble de ces poils (au pluriel) ${ }^{3}$.

Il en va de même dans des dictionnaires plus anciens, comme celui de Trévoux (paru entre 1704 et 1771), qui apportent cependant d'autres précisions intéressantes sur les appellations utilisées en médecine de l'époque pour dénommer les cheveux selon le sexe de celui qui les porte ou encore selon la partie de la tête sur laquelle ils poussent.

Cheveu, s.m : Poil, long, fin \& délié qui vient sur la tête des hommes et des femmes. Capillus. Les Médecins font plusieurs distinctions des cheveux \& leur donnent des noms différents, mais

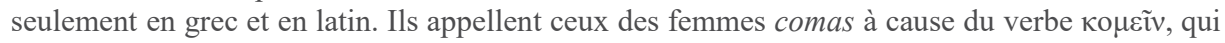
signifie attiffer \& agencer soigneusement; ceux des hommes caefaries a caedeno parce qu'on les coupe souvent; ceux de derrière la tête juba \& crines ; ceux qui pendent derrière oreilles cincinni, c'est-à-dire crepus \& anneles ${ }^{4}$.

Cette différenciation des mots désignant les cheveux n'est jamais entrée dans l'usage commun; qui plus est, avec l'évolution scientifique de la médecine, elle a disparu de la terminologie médicale.

Soulignons par contre l'existence de l'expression en cheveux qui tient compte de la distinction du sexe, en se rapportant uniquement à des femmes. La définition du dictionnaire du CNRTL l'explique : «Se dit d'une femme qui ne porte pas

\footnotetext{
${ }^{2} \mathrm{https} / / / \mathrm{www} . \mathrm{cnrtl}$.fr/definition/cheveux

${ }^{3} \mathrm{https}: / /$ www.larousse.fr/francais/cheveu

${ }^{4}$ https://gallica.bnf.fr/ark:/12148/bpt6k509819/f526.item
} 
d'autre coiffure que ses cheveux $»^{5}$. Rien que par cette précision, «se dit d'une femme », l'expression porte un marquage négatif, compte tenu du fait que ne pas porter de couvre-chef par un homme n'entraîne pas de jugement négatif. La base de données Frantext $^{6}$ apporte quelques exemples littéraires d'emploi de cette expression dont le sens est en général précisé par le contexte. Ainsi Zola l'emploie dans la description d'une femme « en savates et tablier» :

Dehors, le cortège défila. Au coin de la rue Vineuse, une femme en cheveux, les pieds chaussés de savates, pleurait et s'essuyait les joues avec le coin de son tablier.

Émile ZoLA, Une page d'amour (1878 : 1079)

Chez Aragon, une femme en cheveux porte des vêtements pauvres, et comme signe de sa basse condition sociale, elle porte du fil et une aiguille :

Au sein des clameurs. Mademoiselle qui avait disparu un instant ramenait une femme en cheveux, singulière là-dedans, dans ses vêtements pauvres, avec son air gêné... Elle avait encore à la main du fil et une aiguille...

Louis ARAGON, Les Voyageurs de l'impériale (1947: 634)

Camus à son tour insiste sur le comportement peu noble d'une grosse femme en cheveux, parlant fort et gesticulant vivement :

Entre les deux grilles se trouvait un espace de huit à dix mètres qui séparait les visiteurs des prisonniers. J'ai aperçu Marie en face de moi avec sa robe à raies et son visage bruni. De mon côté, il y avait une dizaine de détenus, des arabes pour la plupart. Marie était entourée de mauresques et se trouvait entre deux visiteuses : une petite vieille aux lèvres serrées, habillée de noir, et une grosse femme en cheveux qui parlait très fort avec beaucoup de gestes.

Albert CAMUS, L'Étranger (1942: 1176) ${ }^{7}$

Le sens dévalorisant de l'expression, synonymique de femme du commun, est précisé par Éric Olivier qui écrit :

Qu'aurait-elle dit, si elle avait su que la mère de Christian était une « femme en cheveux » ? À l'époque, les femmes qui ne portaient ni chapeau ni coiffe, n'étaient pas particulièrement estimées. Même les humbles savaient ce que comportait de péjoratif la locution de la femme en cheveux, une femme du commun.

Éric OlLIVIER, L'Orphelin de mer (1982 : 163)

\footnotetext{
${ }^{5}$ https://www.cnrtl.fr/definition/cheveux

${ }^{6} \mathrm{https}: / /$ www.frantext.fr/

${ }^{7}$ Source :

https://www.frantext.fr/repository/frantext/search/concordance?mode=simple\&h=72ae38110d203ce4 61d001ddbe8675a6\&s=eyJ2aWV3Ijoia3dpYyIsImt3aWMiOnsic216ZSI6MTAwLCJkYXRhc2V0cyI 6WyJ3b3JkIl19LCJjb250ZXh0Ijp7InNpemUiOjcwMCwiZGF0YXNldHMiOlsid29yZCJdLCJkZW5 zZSI6ZmFsc2V9LCJ6b29tIjp7InNpemUiOjcwMCwiZm9udFNpemUiOjE1LCJkYXRhc2V0cyI6W yJ3b3JkI10sInR5cGUiOiJkaWFsb2cifSwicGVyUGFnZSI6NTB9
} 


\section{La syntagmatique du mot cheveu}

L'analyse syntagmatique montre la richesse des combinaisons dont le mot cheveu est susceptible. Pour confirmer cette constatation, dans la partie de la recherche qui va suivre, il sera fait recours en premier lieu aux données fournies par le Dictionnaire des cooccurrences Termium plus ${ }^{8}$ et en second lieu aux informations contenues par le $T L F i^{9}$.

\subsection{Les combinaisons adjectivales et verbales du mot cheveu}

Le Dictionnaire des cooccurrences Termium plus en présente deux ensembles : les combinaisons avec un adjectif et avec un verbe, comportant respectivement une centaine et une cinquantaine d'unités.

Parmi les adjectifs qui évoquent les caractéristiques des cheveux, plusieurs se rapportent à leur teinte, à commencer par les lexies traditionnellement réservées à cet usage (roux, auburn, blonds, châtains, bruns, noirs, gris et blancs). Il s'ajoute à cette liste les qualifiants simples qui peuvent être employés dans d'autres collocations (dorés, argentés, platinés, cendrés, flamboyants, rouges, sombres, blanchis), ainsi que des qualifiants issus des noms (filasse, acajou, ébène, aile de corbeau, poivre et sel).

Un autre groupe d'adjectifs se rapporte à l'aspect physique des cheveux et en particulier à leur longueur (longs, coupés courts, tondus), à leur épaisseur (abondants, bouffants, broussailleux, denses, clairsemés, rares), à leur forme (annelés, bouclés, crêpelés, crépus, ondulés, drus, hérissés, hirsutes), à leur aspect (brillants, laineux, soyeux, luisants, lustrés, ternes, poisseux, huileux), à la façon dont ils sont coiffés ou non (défaits, dénoués, désordonnés, dociles, droits, ébouriffés, rebelles, nattés, noués/retenus/roulés/serrés/tirés en chignon, torsadés), etc.

Les cooccurrences du mot cheveux avec les verbes forment à leur tour deux groupes, selon la position de ce mot. En position de Sujet, la lexie en question est accompagnée par les verbes qui expriment le "mouvement» des cheveux (les cheveux croissent, flottent, ondulent, pendent, s'ébouriffent, se dressent, se hérissent, se mêlent, tombent), ainsi que le changement de leur teinte (les cheveux blanchissent, blondissent, foncent, grisonnent). En position d'Objet, le mot suit les verbes qui désignent toutes sortes d'opérations destinées à l'entretien des cheveux (arranger, brosser, coiffer, couper, défaire, démêler, désépaissir, détacher, ébouriffer, effiler, friser, lisser, lustrer, mettre en forme, natter, (dé)nouer, (se) peigner, (se) laver, raccourcir, (se faire) rafraîchir, rattacher, relever, rouler, s'arranger, se (faire) couper, se décolorer, se teindre, tailler, (se faire) tondre), ainsi que ceux qui se rapportent à la «vie » du cheveu (laisser pousser, perdre les cheveux).

\footnotetext{
${ }^{8}$ https://www.btb.termiumplus.gc.ca/

${ }^{9}$ http://atilf.atilf.fr/
} 


\subsection{Les figements construits avec le mot cheveu}

L'étude des locutions groupées dans l'entrée cheveu du TLFi permet de constater qu'elles constituent plusieurs ensembles selon la valeur de cette lexie qui est mise en relief.

Ainsi le premier groupe est formé par les expressions dans lesquelles l'image des cheveux est pour ainsi dire globale, comme c'est le cas de la locution descriptive cheveux au vent qui fait allusion à des cheveux en mouvement, grâce au vent qui souffle.

Les expressions comme se prendre aux cheveux, se tirer les cheveux, $s$ 'empoigner par les cheveux renvoient à la situation d'une querelle qui mène parfois à en venir aux mains - leur cible étant parfois les cheveux de l'adversaire.

À son tour l'expression s'arracher les cheveux, expression qui se rapporte à un profond désespoir, est motivée par l'image du pleureur ou de la pleureuse, personne dont la profession consiste à se lamenter sur la mort de quelqu'un, qui semble survivre encore de nos jours de manière localisée dans certains pays méditerranéens, d'Afrique ou du Moyen-Orient. Proche par le sens, la locution se faire des cheveux ou se faire des cheveux blancs indique la situation où le chagrin est très important : l'expérience humaine apprend que parfois le désespoir fait blanchir les cheveux.

Le second groupe d'expressions est bâti sur l'un des traits spécifiques du mot cheveu, à savoir sa finesse, qui est notamment à la base de la locution comparative mince, fin comme un cheveu. La même valeur de cheveu, avec l'insistance sur une très petite distance qui sépare deux faits, est mise en exergue dans les expressions figées à un cheveu près, il s'en est fallu d'un cheveu que, il s'en est fallu de l'épaisseur d'un cheveu, manquer, rater d'un cheveu. Le même sens de finesse extrême est à la base de couper les cheveux en quatre, locution désignant le fait de subtiliser à l'excès. L'idée de très petite quantité se retrouve aussi dans ne pas vieillir d'un cheveu et dans toucher, ne pas toucher un (seul) cheveu de la tête de $q q n$, ainsi que de la délicatesse dans ne tenir qu'à un cheveu, mais aussi dans tiré(e) par les cheveux.

Dans le troisième groupe, les locutions font appel à la situation où un cheveu peut gêner par sa présence néfaste. C'est le cas de venir, arriver, tomber comme un cheveu sur la soupe, autrement dit arriver à contretemps ou sans aucun propos. Il en est de même dans l'expression avoir un cheveu sur la langue qui désigne le zézaiement.

\section{La sémantique du mot cheveu}

Analysé du point de vue sémantique, le mot cheveu se présente comme une unité polysémique, comme la partie majeure du lexique commun, la polysémie constituant « un trait constitutif de toute langue naturelle » (LEHMANN \& MARTINBerthet, 2000: 65). C'est aussi un mot qui possède un grand nombre de synonymes, surtout en ce qui concerne des variantes non standard. 


\section{La polysémie du mot cheveu}

Le rang qu'occupe le concept cheveu dans la cognition se trouve sans doute confirmé par sa polysémie, à part sa signification première. Ainsi Théophile Gauthier évoque dans Mademoiselle de Maupin (1836, p. 138) les « blonds cheveux des saules du rivage », et Honoré de Balzac les «cheveux des saules, de l'algue, du sainfoin » dans Le Lys dans la vallée (1836, p. 121), en désignant de cette façon les branches retombantes de certaines plantes qui ressemblent à une chevelure. La lexie cheveu s'emploie par analogie de forme aussi pour évoquer une fêlure d'une faïence ou d'une porcelaine. Le blog the-et-ceramique apporte une précision à ce propos :

Une autre trace d'usure, qui résulte d'un accident puisque engendrée par un choc la plupart du temps, est le (sic !) fêle. Lorsqu'il est très fin, il prend le nom de «cheveu » mais il s'agit toujours d'un fêle, d'une fêlure. ${ }^{10}$

L'analogie de forme et/ou de finesse est à la base aussi de plusieurs termes composés autour de la tête cheveu(x), essentiellement dans le domaine de la botanique où ils relèvent du registre populaire. Il est ainsi question notamment des appellations comme d'un côté cheveux de la Vierge ou cheveux de la Bonne Dame et d'un autre côté cheveux de Vénus, qui malgré leur apparente contradiction, se rapportent à la même plante dont le nom standard est clématite. À côté de ces noms à connotation positive qui leur est pourvue par le génitif déterminatif (respectivement la Vierge et Vénus), quelques autres termes composés avec le mot cheveux semblent avoir une coloration neutre, comme c'est le cas de la lexie cheveux d'évêque, synonyme populaire de raiponce, qui fait probablement allusion à la couleur des fleurs de la plante, tirant sur le violet. D'autres dénominations de ce type présentent à leur tour une nuance négative, comme cheveux du diable, synonyme de cuscute. Cette plante parasite peut détruire entièrement les champs de luzerne, mais elle s'attaque également à des légumes (carotte, pomme de terre...), à des arbres (acacia, olivier...) et à des fleurs (dahlia, pétunia...) : il n'est pas étonnant que l'imaginaire populaire y ait vu une présence démoniaque. Or le complément du mot cheveux peut indiquer aussi le milieu naturel de la plante, comme c'est le cas de cheveux de mer, nom populaire des varechs filamenteux, algues très communes, appelées aussi cheveux de sirène.

Il est à noter que le nom cheveux de Vénus existe aussi en minéralogie où il indique « les filaments dorés que forme le rutile dans le quartz hyalin $»^{11}$. Quant au terme cheveux de la Vierge, il est intéressant de constater qu'en entomologie c'est un autre terme pour désigner des fils de la Vierge, à savoir les fils d'araignée des champs. Le terme est cité par Charles Guérin dans Le Couur solitaire:

C'est un des derniers soirs de septembre ;

la brise promène sur les champs les cheveux de la vierge

Charles GUÉRIN, Le Cœur solitaire (1904 : 45)

\footnotetext{
${ }^{10} \mathrm{http}: / /$ the-et-ceramique.blogspot.com/2014/06/porcelaine-les-traces-dusure.html

${ }^{11} \mathrm{https} / / / \mathrm{cnrtl}$.fr/definition/cheveu
} 
À part l'histoire naturelle, différentes technologies ont aussi exploité l'analogie avec le cheveu en appelant diverses entités. Dans le métier de la céramique, il est notamment question de cheveu d'or, terme qui s'applique à « un filet d'or encadrant une pièce de porcelaine $\rangle^{12}$. Dans l'art culinaire et l'art de la décoration, le terme cheveu d'ange désigne, respectivement « une vermicelle très fine ou des tranches d'orange, de cédrat ou de citron, confites et coupées en lanières minces » et « des guirlandes d'arbre de Noël $»^{13}$.

\section{La synonymie du mot cheveu}

Il est bien connu que plus un concept est fréquent, plus il engendre des appellations synonymiques. Or si le mot cheveu est énuméré parmi les 1500 mots les plus fréquents de la langue française (il est question d'une liste constituée par Étienne Brunet ${ }^{14}$, basée sur des extraits de textes de la langue écrite des $\mathrm{XIX}^{\mathrm{e}}$ et $\mathrm{XX}^{\mathrm{e}}$ siècles), il y occupe une place intermédiaire avec ses 3704 apparitions, par rapport au plus fréquent des substantifs, à savoir le mot homme qui est noté 33202 fois et le moins fréquent, le mot gré apparaissant 491 fois. De même, le concept de cheveu n'appelle pas de tabou en français, ce qui neutralise une autre raison qui pourrait favoriser une abondance de synonymes (comme c'est le cas par exemple de la créativité linguistique dans le vocabulaire érotique, cf. Pierre GUIRAUD, Dictionnaire érotique, 1978).

C'est pour ces raisons probablement que les dictionnaires ne présentent que des listes relativement restreintes de synonymes du mot étudié. Sans doute en accord avec le principe selon lequel la synonymie absolue n'existe guère (cf. par exemple LEHMANN \& MARTIN-BERHET, 1998 : 54), souvent elles comprennent des lexies relevant du champ notionnel de cheveu, dont l'équivalence sémantique n'est que très approximative. Ainsi les mots perruque et postiche indiqués comme synonymes de cheveu par certains dictionnaires de synonymes ${ }^{15}$ ne le sont qu'à un niveau très faible : en effet, la différence se situe au niveau du sème /+artificiel/ qui concerne les deux premiers mots.

Quant à la lexie chevelure, sa proximité avec le nom cheveux compris comme « ensemble des cheveux » ${ }^{16}$ semble être la plus élevée, alors que dans le cas de coiffure, même si le mot renvoie aussi à un ensemble de cheveux, l'accent est mis sur « arrangement des cheveux; manière dont ils sont coiffés $»^{17}$.

Le mot chignon à son tour ne désigne qu'un type de coiffure et il doit être considéré comme holonyme de cheveu, vu qu'un chignon se compose de cheveux. Notons cependant que l'expression se prendre aux chignons est synonymique de se prendre aux cheveux (et de se prendre à la crinière), toutes les trois définies comme « se battre », la seule différence étant d'ordre stylistique.

\footnotetext{
$12 \mathrm{https} / / / \mathrm{cnrtl} . \mathrm{fr} /$ definition/cheveu

${ }^{13} \mathrm{https} / / / \mathrm{cnrtl}$.fr/definition/cheveu

${ }^{14} \mathrm{https}: / /$ cache.media.eduscol.education.fr/

$15 \mathrm{http}: / /$ www.synonymo.fr/

${ }^{16} \mathrm{https} / / / \mathrm{www} . \mathrm{cnrtl}$.fr/definition/chevelure

${ }^{17} \mathrm{https} / / /$ www.cnrtl.fr/definition/coiffure
} 
Quelques autres lexies, comme toison ( chevelure épaisse et laineuse $»^{18}$ ), boucles ( la chevelure $»^{19}$ ) et casque ( coiffure de femme enserrant la tête à la manière d'un casque »), tout en se rapportant à une masse de cheveux, indiquent cependant leur aspect particulier. Le mot crinière («chevelure généralement abondante et hirsute ») s'ajoute à ce groupe en évoquant une chevelure abondante, par analogie à un ensemble de poils d'un animal (ex. crinière de lion, crinière de cheval). Dans le cas de la tignasse, il est question en plus de cheveux mal entretenus, l'étymologie de tignasse renvoyant à teigne ${ }^{20}$.

Viennent enfin les synonymes informels, parmi lesquels le dictionnaire du CNRTL note les lexies du type de tifs (avec une variante orthographique tiffes), douilles, persil, cresson, gazon, mouron, crayons. La première parmi elles, les tifs, dont on signale une origine obscure («Peut-être dér. De tifer, en usage dans quelques dialectes aux sens de 'parer, orner, habiller, arranger', et en particulier en wallon tiffé 'coiffer' ») est la seule qui n'aurait pas d'origine métaphorique. Ses plus anciennes attestations datent des années $80 \mathrm{du} \mathrm{XIX}^{\mathrm{e}}$ siècle, or selon le dictionnaire en ligne $B o b$, le mot n'a jamais disparu de l'usage. Argotique d'abord, ce dont témoigne sa présence (sous forme tiffe) dans Parisismen, le dictionnaire bilingue de Césaire Vilatte (1884 : 217), il est passé au XX $X^{\mathrm{e}}$ siècle plutôt dans le style populaire, comme le montre l'exemple ci-dessous, relevé chez Céline :

Ma mère raconte pas non plus comment qu'il la trimbalait, Auguste, par les tiffes, à travers l'arrière-boutique.

CÉLINE Mort à crédit (1936 : 45)

Il est intéressant enfin de noter une mode des dernières années d'exploiter le mot tifs dans un grand nombre de noms de salons de coiffure. De par sa forme, il se prête bien à des jeux de mots comme Cap'Tif ou Créa'Tif et autres. Comme le rapporte Le Monde du 29.11.2016, sur 22094 salons recensés dans la base de données, le palmarès du nom le plus répandu revenait à la forme "Créa Tif », nom dont étaient à l'époque baptisés 134 salons.

Parmi les synonymes non standard de cheveux, énumérons ceux, comme persil, cresson, mouron qui évoquent la même image d'une plante potagère à petites feuilles très denses. Il est question dans ce cas d'une sorte de métaphore filée qui consiste en l'apparition d'un réseau lexical dont les éléments résultent d'une transformation sémantique recourant à des comparés proches (même sans opérer sur plusieurs phrases du même texte, critère définissant la métaphore filée selon (RoBrieUX, 2000 : 53)). Les trois mots semblent fonctionner dans le sens de cheveux dès la fin du XIX ${ }^{\mathrm{e}}$ siècle. Ainsi persil est noté dans le Dictionnaire argotfrançais et français-argot de Georges Delesalle qui l'exemplifie par la phrase «Il n'a plus de persil sur sa tête de veau » (1896, p. 211). Pareil pour le mot cresson, figurant dans le même dictionnaire avec comme exemple «Il n'a plus de cresson

${ }^{18} \mathrm{https}: / /$ www.cnrtl.fr/definition/toison

$19 \mathrm{https}: / / \mathrm{www} . \mathrm{cnrtl} . \mathrm{fr} / \mathrm{definition/boucles}$

${ }^{20} \mathrm{https} / /$ www.cnrtl.fr/definition/tignasse 
sur le caillou (1896: 81). Le mot plumes dans Perdre ses plumes est attesté aussi par Delesalle (1896: 222).

Une autre représentation est à la base de la métaphore gazon, évoquant sans doute les cheveux courts. Le mot marqué dans le dictionnaire du CNRTL comme familier et vieilli est attesté par des exemples du XIX ${ }^{\mathrm{e}}$ siècle, notamment cette description de l'Histoire de ma vie de Georges SAND : "C'était un vieux garçon surmonté d'un gazon jaunâtre » (1855: 417). Le nom pelouse véhiculant la même image de cheveux très courts est cité à son tour par le dictionnaire Bob qui signale que ses emplois sont très rares.

Le mot crayons constitue un autre nom métaphorique de cheveux, attesté à partir des années 1950, notamment chez Le Breton :

Léa [...] refoula une mèche de ses crayons qui lui retombait sur l'œil.

LE BRETON, Razzia (1954, p. 132)

Un autre exemple rapporté dans Bob, venant de la même époque, montre l'emploi du mot vermicels (pour vermicelles) dans le sens de cheveux :

Milou me montre une silhouette blanche. Une gertrude grande, avec des vermicels jusqu'aux reins. Là on a notre chance.

Zep CASSINI (Mollo sur la joncaille, 1955)

Quant à la lexie douilles, les dictionnaires ne sont pas d'accord sur son étymologie. Le CNRTL indique son origine métaphorique comme peu probable, au profit de l'hypothèse de la dérivation régressive à partir de l'adjectif substantivé douillet 'cheveu', fonctionnant en ce sens au XVIII siècle $^{21}$. La plus ancienne attestation du mot proviendrait du Glossaire argotique des mots employés au bagne de Brest (1821) 22 dont provient l'exemple « Le Cardeuil a fait faucher les douilles de tous les amis », rapporté par le dictionnaire en ligne $B o b^{23}$, mais d'autres citations montrent la vitalité de cette lexie encore de nos jours. Elle s'emploie dans plusieurs entourages syntagmatiques, comme: coupe de douilles; se faire couper les douilles; faucher les douilles; rogner les douilles; faire les douilles, etc. Notons aussi les variantes du mot douilles avec le même sens : douillards, douillure et douillets.

Notons encore la forme verlanisée du mot cheveu, à savoir veuch, qui est suffisamment populaire pour apparaître dans une marque de produits pour les soins de cheveux : veuch.co.

Cette brève revue des synonymes informels de cheveu(x) montre leur nombre relativement important, quoique la liste présentée ne soit sans doute pas complète. En effet, le dictionnaire $B o b$ apporte encore d'autres termes, dont l'emploi semble cependant plus rare, notamment : baguettes de tambour, balançoire à poux, bolins,

\footnotetext{
${ }^{21} \mathrm{https}: / / w w w . c n r t l . f r / d e f i n i t i o n / d o u i l l e s$

${ }^{22}$ AnSIAUME Louis (1821), Glossaire argotique des mots employés au bagne de Brest-Argot en usage au bagne de Brest et connu des voleurs de toutes les provinces françaises, manuscrit de la main d'un juge de Chartres, Bibliothèque municipale de Rouen (fonds Montbret).

${ }^{23} \mathrm{https} / / /$ www.languefrancaise.net/Bob/136
} 
brigeants, choucroute, doulots, écailles, filasse, filots, ronces, roseaux, spagues, tigne, tignon, tubes, etc ${ }^{24}$.

Quelques mots et expressions imagées évoquent à leur tour les cheveux blancs et/ou gris : douilles savonnées, filasse savonnée, marguerites, poivre et sel.

Une place à part est occupée par contre par les expressions désignant la calvitie et/ou la perte de cheveux, à commencer par la formule ironique avoir la tête qui dépasse les cheveux. En voici d'autres: n'avoir plus de cressons (de mousse, un poil) sur le caillou (sur la fontaine), être déplumé, ne plus avoir de fil sur la bobine, ne plus avoir de paille sur le tabouret, pas de chapelure sur le jambonneau, perdre ses plumes, plus d'alfa sur les hauts plateaux, plus de gazon sur la fontaine, se dégazonner, se déplumer, tête de veau.

\section{En guise de conclusion}

En parlant du corps humain, il est sans doute banal de constater que certaines de ses parties ou éléments sont vitaux, par exemple la tête, alors qu'il est possible de vivre sans d'autres, comme c'est le cas des cheveux. Or cette distinction physiologique mise à part, il est montré par la langue, plus particulièrement son lexique, l'importance que cet élément véhicule pour l'homme du point de vue socioculturel. Elle se reflète d'un côté par le nombre de mots et d'expressions évoquant les cheveux (y compris dans les variantes non standard du français), leur présence ou absence, leurs formes et leurs couleurs. De l'autre côté, ce qui en témoigne c'est aussi le statut du concept «cheveu» exploité dans de nombreux mots composés relevant de terminologies spécialisées, ainsi que dans les locutions figées insistant sur différents traits typiques de l'entité en question.

La prolifération d'emplois à travers lesquels le mot cheveu apparaît dans le discours constitue sans doute la preuve de son statut de mot incontournable, comparable à la valeur que l'entité même revêt dans le monde extralinguistique.

\section{BIBLIOGRAPHIE}

DARDOUR Jean-Claude (1990), Calvitie et symbolique des cheveux chez l'homme, La Revue de deux Mondes, https://www.revuedesdeuxmondes.fr/wpcontent/uploads/2016/11/84c0aa1969deb03aaeabfea56b7aab11.pdf

DELESALLE Georges (1896), Dictionnaire Argot-Français \& français-Argot, Paris, Paul Ollendorf, Éditeur.

CHRÉTIEN Jean-Louis (2005), Chapitre IX. De la chevelure, in : CHRÉTIEN JeanLouis (éd.), Symbolique du corps. La tradition chrétienne du Cantique des Cantiques, Paris, Presses Universitaires de France, p. 183-200. URL: https://www.cairn.info/symbolique-du-corps--9782130549864-page-183.htm GUIRAUD Pierre (1978), Dictionnaire érotique, Paris, Payot.

${ }^{24} \mathrm{https}: / / \mathrm{www}$. languefrancaise.net/?n=Bob.Introduction\&action $=$ search \&q=cheveu 
KOCH Peter (2005), Aspects cognitifs d'une typologie lexicale synchronique. Les hiérarchies conceptuelles en français et dans d'autres langues, Langue française 145, p. 11-33, https://www.persee.fr/doc/lfr_00238368_2005_num_145_1_6624

LEHMANN Alise, MARTIN-BERTHET Françoise (2000), Introduction à la lexicologie. Sémantique et morphologie, Paris, Nathan.

MIRANDE Yves (2008), Le retour du romantique. La chevelure comme détecteur symbolique, Sociétés 102, p. 61-68. https://www.cairn.info/revue-societes2008-4-page-61.htm

ROBRIEUX Jean-Jacques (2000), Rhétorique et argumentation, $2^{\mathrm{e}}$ édition, Paris, Nathan.

VILATTE Césaire (1884), Parisismen, Alphabetisch geordnete Sammlung der eigenartigen Ausdrucksweisen des Pariser Argot. Ein Supplement zu allen französisch-deutschen Wörterbüchern, Berlin, Langenscheidt.

\section{DICTIONNAIRES ET BASES DE DONNÉES CONSULTÉS :}

http://atilf.atilf.fr/

https://www.btb.termiumplus.gc.ca/

https://www.cnrtl.fr

https://www.cnrtl.fr/dictionnaires/anciens/trevoux/lorraine.php

https://www.frantext.fr

https://www.languefrancaise.net/Bob

https://www.larousse.fr

http://www.synonymo.fr/ 